\title{
USE OF SHAPE MEMORY ALLOY FOR A PILOT STAGE ACTUATOR OF A WATER HYDRAULIC CONTROL VALVE
}

\author{
Marco Vuorisalo and Tapio Virvalo \\ Institute of Hydraulics and Automation Tampere University of Technology \\ P.O.Box 589 FIN-33101 TAMPERE FINLAND \\ (E-mail: marco.vuorisalo@tut.fi)
}

\begin{abstract}
Shape memory alloys have many unique properties. These alloys can be plastically deformed with moderate forces at low temperature, and upon exposure to some higher temperature the alloys will return to its original shape prior to the deformation with more than decade higher force. The power density of shape memory alloys is high and the inactivity in harsh environments makes them enticing choice for valve actuators. Still the shape memory alloy has one disadvantage that makes valve actuator appliances hard to find. The transformation between different states of shape memory alloy is proportional to the energy supplied when the alloy is heated but the cooling is only proportional to the heat transfer ratio between the alloy and environment. Using pair of thin shape memory alloy fibers replacing a torque motor of a traditional nozzle flapper servovalve has been studied. The studied flapper actuator consists of two SMA-fibers arranged in antagonist fashion. When one fiber serves as an actuator the other one serves as a spring. Both fibers are immersed in water in order to increase heat transfer rate. The performance of developed shape memory fiber actuator has been simulated. Simulations have been verified by comparing simulation results to experimental tests. Based on experimental tests of actuator the performance of shape memory fiber actuated nozzle flapper pilot stage has been simulated with verified valve model. The attained overall performance of a 2-stage 4-way spool valve with the developed pilot stage has been discussed.
\end{abstract}

\section{KEY WORDS}

Shape memory, Pilot stage actuator

\section{INTRODUCTION}

The shape memory effect was first discovered by the Swedish physicist Arne Olander in 1932 using an alloy of gold and cadmium. After almost thirty years U.S.
Naval ordinance Laboratory discovered the shape memory effect in nickel titanium alloy and named it Nitinol. Still not until the early 1970's several commercially available products appeared that implemented shape memory alloys (SMA). 
In SMA's are two different features that can be used. These are the superelasticity and the shape memory effect (SME). SME can be used actively or passively. The passive way is to let the SMA react to the environment temperature changes like in thermostats. The active way is to use Joule heating so SME can be controlled electrically.

Depending on the temperature SMA has three different phases. Phases are called the martensite, the R-phase and the austenite. The R-phase has similar macroscopic properties as the martensite, but the material properties differ. In some cases the R-phase is masked in martensite phase. Reason why the R-phase is interesting is that the $\mathrm{R}$-phase-the austenite transformations exhibits narrow hysteresis and negligible fatique effects. In this study however the R-phase is not considered, but phase change is supposed from the martensite to the austenite and back. Usually the memory effect in the SMA is one-way. That is, upon cooling, a shape memory alloy does not undergo any shape change, even though the structure changes to the martensite. When the martensite is strained up to several percent however, that strain is retained until the material is heated, at which time shape recovery occurs. Upon re-cooling, the material does not spontaneously change shape, but must be deliberately strained if shape recovery is again desired [10].

\section{IMPROVING THE RESPONSE OF SMA ACTUATORS}

Traditionally shape memory actuators have relationally slow response. Reason for slow response is not the transformation rate. The transformation rate is much faster than the practical response speed of SMA actuators. In fact since the practical response speed of SMA actuators is limited by the rate of heating and cooling, visco-elastic effect due to transformation is negligible in this case [7]. Heating rate increasing is straightforward by increasing electric power supply also heating rate improve. Increasing the cooling rate is harder so it is the main target when improving the SMA response. Changing from free convection to forced convection, using fixed heat sink or immersing SMA to a liquid increase cooling rate. All listed methods increase cooling rate but same time also heating power demand grows so the coefficient of efficiency decreases. To avoid this various sophisticated cooling methods have been introduced including use of Peltier-cooler and mobile heat sink [8].

The coefficient of efficiency is always low in macro sized SMA-actuator. The energy efficiency of SMA is theoretically restricted to approximately $10 \%$ by the
Carnot efficiency cycle. Efficiency is often less than 1\% in practical applications, since the driving principle of the actuator can be considered as a heat engine operating at low temperatures [3]. The very poor efficiency is the reason why fixed heat sinks or immersion is hardly ever used with SMA-actuators. As a rule of thumb when SMA-fiber is immersed in water it needs 20 times more heating power than in still air.

If SMA is used as pilot valve actuator the most compact way to increase response speed is to immerse the actuator in the pressure medium. In case of nozzle-flapper pilot stage this is simply done by including both actuators and the flapper in the same space where tank flow from nozzles immerses the actuator.

\section{SMA-ACTUATORS AS PILOT VALVE ACTUATORS}

Energy efficiency with the SMA is poor but the situation in not so severe if the SMA is used as a pilot stage actuator because of hydraulic amplification. Pilot stage power consumption is minor compared to overall power transmission.

SMA-actuators have unique properties compared to any other type actuator. They have very high power to weight ratio and excellent environment compatibility. These features combined together makes possible to approach the valve actuator problem from whole new point of view. Traditional nozzle flapper-pilot valve actuators have few basic parts flapper, torque tube, armature, control coils and permanent magnet (Figure 1)
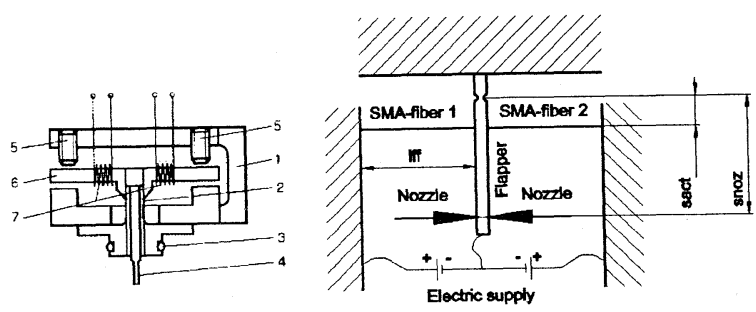

Figure $1 \mathrm{~A}$ dry torque motor actuator [9] and an example of a nozzle flapper pilot stage construction with SMAactuators.

If same pilot valve actuator is constructed with SMAfibers it can be composed of three parts, SMA-fibers and flapper (Figure 1). Because most SMA-fibers have only one-way memory pre-stress have to be applied to strain the fibers while assemblage. When one fiber is heated it recovers its unstrained shape and the other fiber serves as spring. Because the flapper is fixed between the actuators 
a bending motion to the flapper is attained. Upon recooling the unheated fiber and flapper strain heated fiber back to its prior length

\section{SMA-FIBER SIMULATION}

\section{Thermo-mechanical behavior}

Modeling of the thermo-mechanical behavior of SMAfiber is based on the work of Tanaka [2] with few simplifications.

$$
\dot{\sigma}=D \dot{\varepsilon}+\Theta \dot{T}+\Omega \dot{\xi}
$$

where $\sigma$ is the second Piola-Kirchhoff stress, $\varepsilon$ the green strain, $T$ the temperature and $\xi$ the martensite ratio. The material parameters $D, \Theta$ and $\Omega$ are the elastic modulus, the thermoelastic tensor and the transformation tensor. These material parameters are assumed constant. The higher order terms of Green strain and second PiolaKirchhoff stress are assumed negligible too.

In constrained case like antagonist set of actuators strain $\varepsilon$ can be set to zero so Eq. (1) becomes

$$
\dot{\sigma}=\Theta \dot{T}+\Omega \dot{\xi}
$$

The martensitic ratio $\xi$ is an internal variable that is used to account for the phase change in of the SMA. It is the ratio of martensite in alloy and varies from complete martensite $\xi=1$ to complete austenite $\xi=0$. The ratio is dependent on the applied stress and temperature. Modeling of martensite ratio is difficult because besides to acting variable also hysteresis is present. Still in antagonist actuator case hysteresis can be ignored because only one actuator at a time drives the system. So the system response will then always be determined by the martensite-austenite part of hysteresis curve of the alloy. As a large portion of the martensite ratio transformation is linear, a linear relation can be used to capture their relationship during the heating process [3].

$$
\xi_{M \rightarrow A}= \begin{cases}1 & T<c_{m} \sigma+A_{s 0} \\ \frac{c_{m} \sigma+A_{f 0}-T}{A_{f 0}-A_{s 0}} & c_{m} \sigma+A_{f 0} \geq T \geq c_{m} \sigma+A_{s 0} \\ 0 & T>c_{m} \sigma+A_{f 0}\end{cases}
$$

Where $A_{f 0}$ and $A_{s 0}$ are the stress free transformation temperatures and $c_{m}$ is the inverse of the stress rate $1 / c_{m}$. In above determined temperature and stress range the derivative of martensite ratio becomes

$$
\dot{\xi}=K_{m} c_{m} \dot{\sigma}-K_{m} \dot{T}
$$

where the constant

$$
K_{m}=\frac{1}{A_{f 0}-A_{s 0}}
$$

\section{Heat transfer and stored energy balance}

Assuming temperature variations in SMA-fiber negligible the heat transfer rate to or from the SMA becomes equal to the energy stored in the SMA. Method is called lumped heat capacity.

$$
\dot{Q}=\dot{E}
$$

Where $Q$ is the heat transfer rate to or from the SMA and $E$ is the stored energy in the SMA.

Energy is stored in the SMA by increasing the temperature and through the phase transformation.

$$
\dot{E}=m_{f} c_{p} \dot{T}-m_{f} L_{a} \dot{\xi}
$$

Where $m_{f}$ is the mass of the fiber, $c_{p}$ is the specific heat, and the $L_{a}$ is the latent heat of the transformation.

The Joule heating and convection cooling determine the heat transfer rate. Conduction is assumed negligible.

$$
\dot{Q}=I_{f}(t)^{2} R_{f}-h a_{r}\left(T-T_{0}\right)
$$

where $a_{r}$ is the surface area of the fiber, $T_{0}$ the ambient temperature, $I_{f}$ the fiber input current, $R_{f}$ the fiber resistance and $h$ the convection coefficient.

So the energy balance based on Eq. (6) becomes

$$
I_{f}(t)^{2} R_{f}-h a_{r}\left(T-T_{0}\right)=m_{f} c_{p} \dot{T}-m_{f} L_{a} \dot{\xi}
$$

Based on Eq.(2), Eq. (4) and Eq. (9) a simulation model from the input current to the output force can be combined.

\section{Parameters and constants}

Both phase transformation tensor $\Omega$ and thermoelastic tensor $\Theta$ are assumed constant. Transformation tensor for constrained recovery case is between $600-800 \mathrm{MPa}$ [3]. Values of thermoelastic tensor varies between $0.1-0.5$ $\mathrm{MPa} / \mathrm{K}$ depending on the source of information. Stress rate $1 / c_{m}$ depends on the on composition. Values form $3-$ $20 \mathrm{MPa} / \mathrm{K}$ are stated [4]. Specific heat $c_{p}$ and latent heat of transformation $L_{a}$ have large variation depending on the alloy composition. Specific heat varies between 450 $620 \mathrm{~J} / \mathrm{Kg} \mathrm{K}$ and latent heat between $19000-32000 \mathrm{~J} /$ $\mathrm{Kg}$ [5].The convection coefficient $h$ is highly application dependent. Beside convection areas acting factors are 
type of fluid and flow rate of actuator surrounding fluid. Boiling of fluid still increases convection that is more likely occur in case of water and SMA-fiber. Free convection in water is in range $800-900 \mathrm{~W} / \mathrm{m}^{2}$. If convection is forced coefficient easily increases 4 times and if boiling occurs values of $10000 \mathrm{~W} / \mathrm{m}^{2}$ are introduced [6]. The numerical values of simulation parameters in Table 1 are mainly from literature [3], [5], [11]. The used latent heat term is exceptionally high and reason for that is the defect of the modeling of the martensite ratio.

Table 1 Parameter values of SMA-fiber

\begin{tabular}{|c|c|c|c|}
\hline Parameters & Symbol & Value & Units \\
\hline $\begin{array}{c}\text { Phase } \\
\text { transformation } \\
\text { tensor }\end{array}$ & $\Omega$ & $600^{*} 10^{6}$ & $\mathrm{~J} /\left(\mathrm{Kg}^{*} \mathrm{~K}\right)$ \\
\hline $\begin{array}{c}\text { Thermo elastic } \\
\text { tensor }\end{array}$ & $\Theta$ & $0.2^{*} 10^{6}$ & $\mathrm{~Pa} / \mathrm{K}$ \\
\hline Stress rate & $1 / c_{m}$ & $7.14^{*} 10^{6}$ & $\mathrm{~Pa} / \mathrm{K}$ \\
\hline Specific heat & $c_{p}$ & 350 & $\mathrm{~J} /\left(\mathrm{Kg}{ }^{*} \mathrm{~K}\right)$ \\
\hline Latent heat & $L_{a}$ & 56000 & $\mathrm{~J} / \mathrm{Kg}$ \\
\hline $\begin{array}{c}\text { Heat transfer } \\
\text { coefficient }\end{array}$ & $h$ & 1500 & $\begin{array}{c}\mathrm{W} /\left(\mathrm{m}^{2} *\right. \\
\mathrm{K})\end{array}$ \\
\hline $\begin{array}{c}\text { Density } \\
\text { Austenite finish } \\
(\xi=0, \sigma=0)\end{array}$ & $\rho$ & 6450 & $\mathrm{Kg} / \mathrm{m}^{3}$ \\
\hline $\begin{array}{c}\text { Austenite start } \\
(\xi=1, \sigma=0)\end{array}$ & $A_{s 0}$ & 373 & $\mathrm{~K}$ \\
\hline $\begin{array}{c}\text { Intrinsic } \\
\text { resistance }\end{array}$ & $R_{f}$ & 42 & $\Omega / \mathrm{m}$ \\
\hline
\end{tabular}

Table 2 Dimensions of SMA-fiber and clamps

\begin{tabular}{|c|c|c|c|}
\hline $\begin{array}{c}\text { Dimensions and } \\
\text { constants }\end{array}$ & Symbol & Value & Units \\
\hline Length of fiber & $l_{f}$ & $50^{*} 10^{-3}$ & $\mathrm{~m}$ \\
\hline $\begin{array}{c}\text { Effective length } \\
\text { of fiber }\end{array}$ & $e l_{f}$ & $25^{*} 10^{-3}$ & $\mathrm{~m}$ \\
\hline Diameter of fiber & $d_{f}$ & $0.152^{*} 10^{-3}$ & $\mathrm{~m}$ \\
\hline $\begin{array}{c}\text { Equivalent mass } \\
\text { of clamps }\end{array}$ & $m_{c}$ & $3.75^{*} 10^{6}$ & $\mathrm{Kg}$ \\
\hline $\begin{array}{c}\text { Equivalent area of } \\
\text { clamps }\end{array}$ & $A_{c}$ & $20^{*} 10^{-6}$ & $\mathrm{~m}^{2}$ \\
\hline $\begin{array}{c}\text { Specific heat of } \\
\text { steel }\end{array}$ & $c_{s}$ & 500 & $\mathrm{~J} /\left(\mathrm{Kg}^{*} \mathrm{~K}\right)$ \\
\hline Bias stress of fiber & $S_{b}$ & 10000 & $\mathrm{~Pa}$ \\
\hline
\end{tabular}

Simulation model

Various different studies are published of SMA-actuator simulation [3] [7] [11]. None of them can directly apply in case where SMA-fibers are immersed in pressure medium. If actuators are immersed in water the heat convection coefficient is so high that the delay before phase change to the austenite starts have to be considered.

Used thermo-mechanical equations are only valid between the austenite start and austenite finish temperatures corrected with temperature increase with increased stress. In the developed model the validity box (Figure 3) observes the limitation of validation at the low temperature by supplying zero when temperature is below austenite start temperature. The upper limitation is done by setting upper saturation limit of the integrator to the maximum deformation strength of the SMA fiber.

The equivalent mass and area of fiber holding clamps are included in the simulation model because part of the heat transfer ratio becomes of the heat transfer via clamps. Heat transfer via clamps can be avoided with suitable material selection but in this case steel clamps were chosen. The electric conductivity of water is not modeled because the wasted power in water is negligible compared the supplied power.

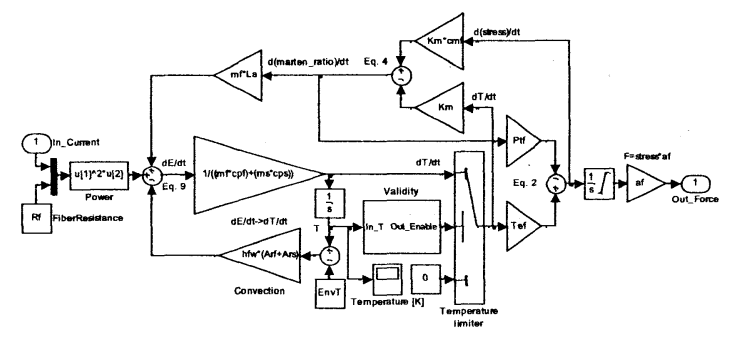

Figure 2 Block diagram of a single SMA-fiber.

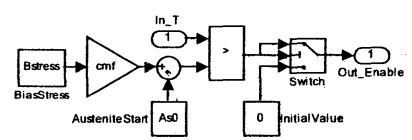

Figure 3 Block diagram of subsystem validity.

In Figure 4 simulated force responses are compared to the measured responses. As can be seen the heating sides of force responses are accurate but the cooling sides are not. The hysteresis of transformation temperatures is not modeled and it makes considerable error in cooling side of the force curves. This is not a concern because the heating sides of the curves dominate in antagonist actuator case. The nonlinear nature of martensite transformation can be seen in both ends of the measured force response curves as well. 


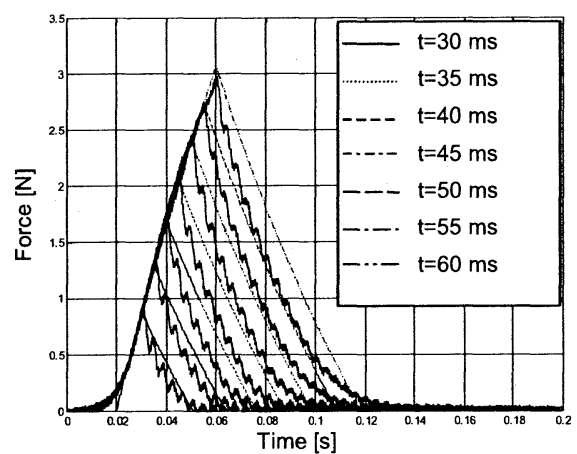

Figure 4 Simulated and measured force response curves of a single SMA-fiber with varying pulse durations.

\section{COMPARISION OF ACTUATORS}

The performance of the SMA-pilot actuator and the torque motor pilot actuator were compared using a simulation model of a nozzle flapper servovalve with pressure feedback. The simulation model was created to develop a low-pressure control valve for water hydraulics [12]. Also in this comparison the used pressure level was $3 \mathrm{MPa}$ and the used pressure medium was water. In the valve model torque motor part is straightforward for example electrical delay is not modeled. Still the model servers as comparison level what can be expected from a pilot actuator.

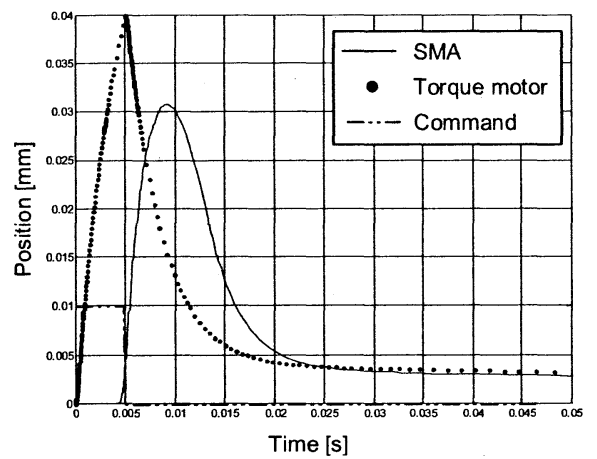

Figure 5 Flapper step response to $5 \mathrm{~ms}$ pulse with torque motor and SMA-fiber actuators.

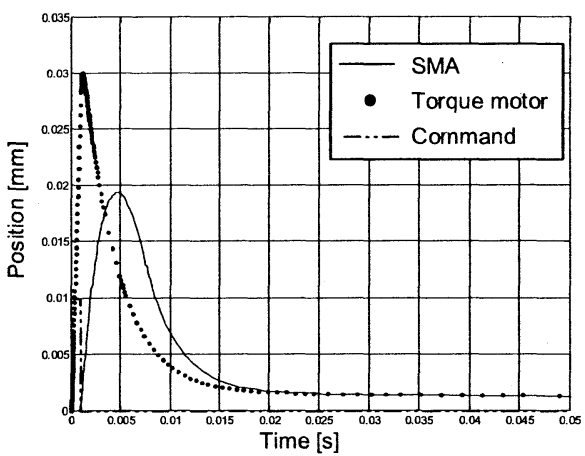

Figure 6 Flapper step response to $1 \mathrm{~ms}$ pulse with torque motor and SMA-fiber actuators.

In Figure 5 and Figure 6 can be seen the flapper response to supply current pulses. In Figure 5 the supply current of torque motor actuator is $40 \%$ of maximum and in Figure 6 supply current is $100 \%$. The corresponding pulse durations are $5 \mathrm{~ms}$ and $1 \mathrm{~ms}$. The supply currents and pulse durations are chosen not to saturate the flapper movement. In SMA-actuated flapper can be seen $5 \mathrm{~ms}$ delay with $5 \mathrm{~ms}$ pulse and $1 \mathrm{~ms}$ delay with $1 \mathrm{~ms}$ pulse. The fiber heating from pressure medium temperature to the austenite start temperature causes this delay. The delay can be avoided by supplying suitable quiescent current to fiber to keep fiber temperature near austenite start temperature. In Figure 5 the flapper step responses of both actuators have similar gradients but in Figure 6 differences becomes visible. Still when the spool responses in Figure 7 and in Figure 8 are observed the difference in gradients are not visible.

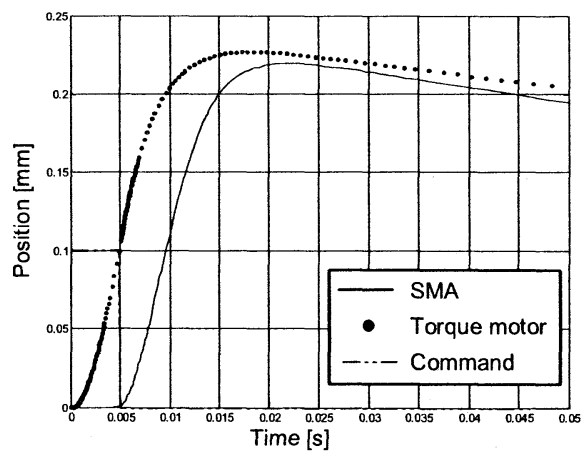

Figure 7 Spool step response to $5 \mathrm{~ms}$ pulse with torque motor and SMA-fiber actuators. 


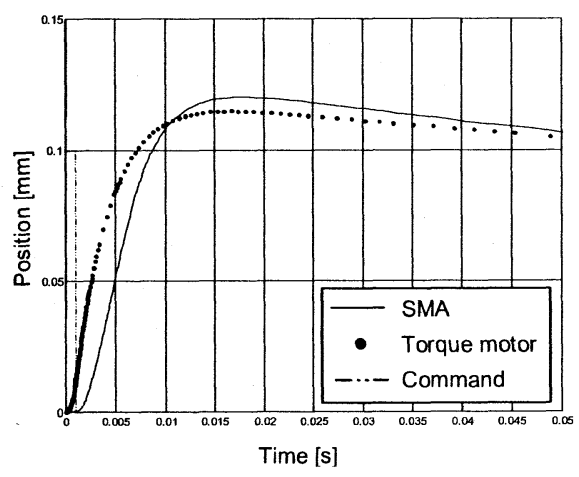

Figure 8 Spool step response to $1 \mathrm{~ms}$ pulse with torque motor and SMA-fiber actuators.

In Figure 9 can be seen the force response of antagonist SMA-actuator pair. Although force response curve is clearly asymmetric the asymmetric nature of pilot stage actuator shows as rounding in the flapper step response curve. But cannot be seen in the spool response curve

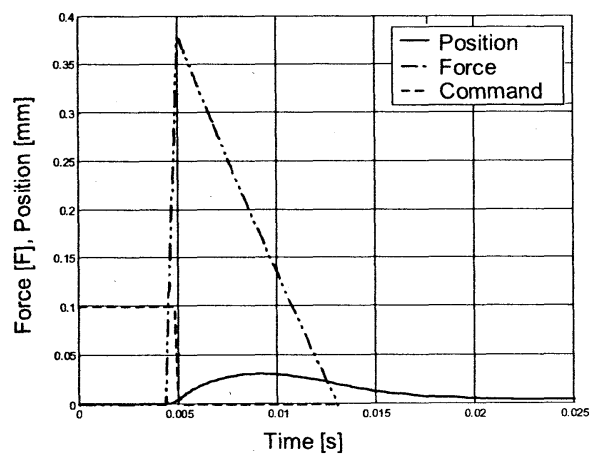

Figure 9 SMA-actuator force and flapper position step response to $5 \mathrm{~ms}$ pulse.

\section{CONCLUSION}

The performance of SMA-fiber actuator is sufficient for a pilot control actuator at least for low-pressure control valve. Torque motor has better performance but if the main spool step responses are compared the spool opening gradients are identical. This leads to conclusion that SMA-fibers are fast enough for this solution both heating and cooling rate are adequate .

The problem of using SMA-fibers is the high power demand. In this case the power demand was higher than necessary because the SMA-actuator fiber was too strong compared to the demanded power. Only tenth of available power of SMA was used and even that could have been smaller if lengths sact and snoz (in Figure 1) have been chosen vise versa. Anyhow heating of SMAfiber needs few amperes power and torque motor needs few milliamperes.

The advantage of SMA-fiber pilot stage is small space demand. If power demand is thousand times higher as well the actuator space demand is thousand times smaller. This makes possible for example make a valve spool where the pilot stage actuator and flapper are inside the spool.

1. Kohl M., Just E., Pflening W., S. Miyazaki, SMA microgripper with integrated antagonism, Sensors and Actuators 83 (2000), pp. 208-213

2. Tanaka K, A Thermomechanical Sketch of Shape Memory Effect: One-dimensional Tensile Behaviour, Res Mechanica 18 (1986), pp.251-263

3. Grant D., Accurate and Rapid Control of Shape Memory Alloy Actuators, Dissertation Thesis, Department of Electrical and Computer Engineering McGill University, 1999

4. http://www.mtm.kuleuven.ac.be/Research/ADAPT/ publicat/ShapeMemory/smatab.htm 2.4.2002

5. Huang W., On the selection of shape memory alloys for actuators, Materials and Design 23 (2002) 11-19.

6. Holman J. P., Heat Transfer, McGraw-Hill, Inc 8th Edition, USA, 1997 ISBN 0-07-114320-3

7. Ikuta K., Tsukamoto M., Hirose S., Mathematical Model and Experimental Verification of Shape Memory Alloy for Designing Micro Actuator.

8. Russell R. A., Gorbet R. B., Improving the Response of SMA Actuators, International Conference on Robotics and Automation, 1995, PP. 2299-2304

9. Lang R., The hydraulic Trainer Volume 2 Proportional and Servo Valve Technology, Mannesmann Rexroth GmbH, 1989

10. Hodgson D. E., Ming H. W., Biermann R. J., http://www.sma-inc.com/SMApaper.html 18.4.2002

11. Reynaerts D., Van Brussel H., Design aspects of shape memory actuators, Mechatornics 8 (1998), pp. 635-656.

12. Vuorisalo, M., Virvalo, T. \& Anttonen, P. 2001. Different types of pilot stages for a water hydraulic control valve. Proceedings of the Fifth International Conference on Fluid Power Transmission and Control (ICFP'2001), China. pp. 435-439. 